

\title{
The Flow Stress of High-Purity Refractory Body-Centred Cubic Metals and its Modification by Atomic Defects
}

A. Seeger

\section{- To cite this version:}

A. Seeger. The Flow Stress of High-Purity Refractory Body-Centred Cubic Metals and its Modification by Atomic Defects. Journal de Physique IV Proceedings, 1995, 05 (C7), pp.C7-45-C7-65. 10.1051/jp4:1995704 . jpa-00253997

\section{HAL Id: jpa-00253997 https://hal.science/jpa-00253997}

Submitted on 1 Jan 1995

HAL is a multi-disciplinary open access archive for the deposit and dissemination of scientific research documents, whether they are published or not. The documents may come from teaching and research institutions in France or abroad, or from public or private research centers.
L'archive ouverte pluridisciplinaire HAL, est destinée au dépôt et à la diffusion de documents scientifiques de niveau recherche, publiés ou non, émanant des établissements d'enseignement et de recherche français ou étrangers, des laboratoires publics ou privés. 


\title{
The Flow Stress of High-Purity Refractory Body-Centred Cubic Metals and its Modification by Atomic Defects
}

\author{
A. Seeger \\ Max-Planck-Institut für Metallforschung, Institut für Physik, Heisenbergstrasse 1, 70569 Stuttgart, \\ Germany
}

\begin{abstract}
The strong temperature and strain-rate dependence of the flow stress of high-purity refractory body-centred cubic metals has been shown to be an intrinsic property and is usually ascribed to a high Peierls barrier of $a_{0}\langle 111\rangle / 2$ screw dislocations. These barriers are overcome by the formation of kink pairs on the screw dislocations. The paper reports on recent, very complete flow-stress data on ultra-high purity Mo crystals obtained by two different experimental techniques and covering the temperature range $4 \mathrm{~K}$ to $460 \mathrm{~K}$. The results are in accord with earlier work of Brunner and Diehl on $\alpha-F e$, who showed that below the so-called knee temperature, $T_{\mathrm{K}}$, three regimes in the temperature variation of the flow-stress should be distinguished. Two of them are fully accounted for by the same glide mechanism, namely elementary glide steps on $\{211\}$ planes. The so-called upper bend separating these two regimes is an inherent feature of the theory of kink-pair formation and does not indicate a change in the glide mechanism. There is, however, strong evidence that the so-called lower bend, separating the range of $\{211\}$ elementary glide steps from the low-temperature flow-stress regime, is due to a change in the glide mechanism. It is argued that at the lower bend the screw-dislocation cores undergo a "first-order phase transition" from a low-temperature configuration that allows glide of a given screw dislocation on any of its three $\{110\}$ glide planes to a high-temperature configuration that can glide only on one definite $\{211\}$ plane. Between $T_{\mathrm{K}}$ and the lower-bend temperature, $\widetilde{T}$, bcc metals may show the unique phenomena of alloy and irradiation softening. With regard to the latter phenomenon, Brunner and Diehl distinguish between "primary" and "secondary" softening. It is shown that alloy softening and the "secondary irradiation softening" of bcc metals may be explained by an "overheating" of the phase transition in the dislocation core. Although the underlying mechanism is not yet fully understood, there is fairly strong evidence that the "primary irradiation softening" is due to the interaction between screw dislocation cores and crowdion self-interstitials.
\end{abstract}

\section{INTRODUCTION}

This paper is a sequel to the author's contribution "Imperfections in Ultra-High-Purity Metals: Past, Present and Future" to the First International Conference on Ultra-high Purity Base Metals [1]. There it was recalled that with very few exceptions (e.g., the electrical or thermal conductivity at ambient or elevated temperatures) the technologically important properties of metals are governed by deviations from the ideal crystal structure. These deviations may either be "chemical imperfections" (e.g., substitutional foreign atoms, not disturbing the crystallographic structure) or "physical imperfections", e.g. dislocations, grain boundaries, vacant sites, etc., not involving foreign atoms (also known as "lattice imperfections"), or a combination of both. Achieving a genuine understanding of the rôles played by these imperfections as well as their properties as individual entities is therefore not only a fascinating task of basic research in solid-state physics but also the key to many applications of metals and alloys.

The field has been developing rapidly since the end of World War II, i.e., for the last five decades. Not only has it had a strong impact on the science and technology of metals and alloys but it has also decisively influenced neighbouring fields. A large number of important experimental tools and theoretical concepts have emerged from the work on imperfections in metals. After an initial period in which some of the key concepts were developed on ionic crystals (cf.[2]), metals became the "trail-blazers" in the study of lattice defects in crystals. The rapid progress in studying and understanding lattice defects in semiconductors and ceramics would hardly have been possible without the foundations laid by the work on metals and alloys. The main features to which metals owe this rôle are

(i) the simplicity of most of their crystal structures, 
(ii) their dominance in the periodic system and thus the wide variations in their imperfection-insensitive properties, which allow us to test the general validity of our theoretical concepts,

(iii) the possibility of producing at least some metals (by no means the majority!) with ultra-high purity and to maintain them in this state during subsequent experimentation,

(iv) the possibility of growing large high-quality (and often also high-purity) single crystals. An outstanding example of items (iii) and (iv) is the metal molybdenum, which will feature prominently in this review.

As outlined in [1], a crucial problem in studying imperfections in any class of solids is that it is very difficult to devise experiments in which we can be sure that only a small number of different types of imperfections or - preferably - just one kind of defect have to be considered. In overcoming this difficulty, ultra-high-purity metals are of key importance. They offer the possibility of establishing experimental conditions under which the influence of foreign atoms ("chemical imperfections") can be disregarded. This gives us the chance to investigate the properties of intrinsic lattice defects and to study, in a subsequent step, their interaction with foreign atoms that have been introduced in a controlled way. (In this context, we deliberately avoid the widely used term "impurities".)

The strategies available for the study of various types of imperfections in ultra-high purity metals have been discussed in detail in the previous paper [1]. There the main emphasis was on intrinsic atomic defects (vacancies, self-interstitials) and on the mechanisms of self-diffusion. The present paper will deal with plastic deformation and, in particular, with the interaction between dislocations and atomic defects.

The plastic deformation of crystals by glide is a field in which defect-defect interactions are of paramount importance. Hardening, be it due to the deformation itself (= work-hardening), or to irradiation, alloying, formation of precipitates, etc., involves the interaction of dislocations either among themselves or with other perturbations of the ideal crystal structure. The strengthening of metals by alloying has been known to mankind for thousands of years. It has not only played a key rôle in the development of the implements and weaponry of the various civilisations, but has also provided us with a vast amount of empirical metallurgical knowledge. A scientific understanding of the alloying effects on the strength and the work-hardening properties of metals, however, became possibie only after experiments on man-made metal single crystals of reasonably good purity could be performed. For the pioneering work in this field, which was carried out within about one decade between 1922 and 1932 in two Kaiser-Wilhelm-Institutes in Berlin (among them the predecessor of the Max-Planck-Institut für Metallforschung) and in Cambridge University the reader is referred to the still very readable account by Schmid and Boas [3].

In the present context it is noteworthy that some of the early basic experiments were performed on materials with quite good purity, e.g., those on cadmium single crystals of "Kahlbaum" quality with $99.996 \%$ purity [4]. A decisive result of the pioneering work on metals single crystals was that in the metals with close-packed [ =hexagonal close-packed (hcp) or face-centred cubic (fcc)] structure the critical shear stress $\sigma_{0}$ (= resolved shear stress at which glide sets in) was not only very low at room temperature (in the above-mentioned "Kahlbaum" cadmium $\sigma_{0}=570 \mathrm{kPa}$ at room temperature, corresponding to a critical shear strain of $3 \cdot 10^{-5}$, or to $10^{-3}$ of the theoretical critical strain $\left.[5,6]\right)$ but that it was not dramatically higher even at liquid-helium temperatures [7].

After earlier work by Prandtl [8], who had in mind the explanation of the internal friction and the elastic aftereffect (now known, following Zener [9], as "anelasticity") and of Dehlinger [10], who invented the concept of "Verhakungen"(= dislocation dipoles) in order to account for the internal stresses of the third kind in deformed metals, it was the desire to understand the low critical shear stress of pure metals that led to the concept of dislocations $[11,13]$.

From the first papers on dislocations - particularly those by Orowan [11] and Polanyi [12] - and, mutatis mutandis, also from those on their predecessors, Prandtl's "Vernier defects"[8] and Dehlinger's "Verhakungen" [10], it is obvious that their authors had realized what in modern language may be 
described as follows. As a consequence of the discreteness of the crystal structure, the energy of a straight dislocation running parallel to a low-index crystallographic direction is a periodic function of its position on its glide plane, with a period that is determined by the crystal structure. This means that such a dislocation may occupy a sequence of energetically favoured positions with separations of the order of magnitude of and commensurate with the lattice parameter. A consequence of this is that a finite resolved shear stress must act on the dislocation in order to move it through an otherwise perfect crystal. From a theoretical point of view a suitable quantitative measure of this is the minimum resolved shear stress $\sigma_{\mathrm{P}}$ required in order to lift a straight dislocation line out of its low-energy valleys in the absence of thermal or quantum-mechanical fluctuations. For a given Burgers vector, $\sigma_{P}$ depends on the crystallographic direction of the dislocation line, i.e., on the dislocation character. Physical intuition indicates that $\sigma_{\mathrm{P}}$ is largest for the most densely packed directions.

As the first serious attempt to calculate $\sigma_{\mathrm{P}}$ was undertaken by R. Peierls [14] (at the suggestion of $E$. Orowan, cf. [15]), we refer to it as Peierls stress, to the corresponding energy barriers as Peierls barriers, and to the valleys of low dislocation energy as Peierls valleys. Peierls' calculation was later improved by Nabarro [16] and by Dietze [17]. For glide on close-packed planes (i.e., for fcc metals and for basal glide in hcp metals) the calculated Peierls stresses came out significantly higher than the measured critical shear stresses.

The debate whether "high" Peierls stresses of the order of magnitude $10^{-4}$ of the shear modulus $M_{\text {sh }}$ are compatible with critical shear stresses that are a power of ten or more smaller has been going on since the Frank-Read milling mechanism [18] was proposed as the dominant mechanism of dislocation multiplication. According to this mechanism - or rather its generalizations, since "pure" Frank-Read sources have only rarely been observed-multiplying dislocations have at some stage to run parallel to close-packed crystallographic directions. There they will encounter the high Peierls barriers mentioned above. In the absence of thermal fluctuations helping to overcome them, i.e., at low temperatures, these barriers should determine the critical shear stresses.

The above-mentioned discrepancy between experiments, e.g. those of Blewitt et al. [19] on the lowtemperature flow stress of copper or the above-mentioned early work on $\mathrm{Cd}$ [7], and theory led some workers in the field to believe that the Peierls stress in the fcc metals and for basal glide in the hcp metals had to be lower by some orders of magnitude than the theoretical estimates. Evidence to the contrary was provided by the present writer when he showed [20,21] that the low-temperature internal-friction maximum in cold-worked fcc metals discovered by Bordoni $[22,23]$ and a related one subsequently found by Niblett and Wilks [24] could be interpreted in terms of the stress-assisted thermally activated overcoming of the Peierls barriers. This interpretation, which has stood the test of time, implies Peierls stresses in the fcc metals of the order of magnitude of $10^{-4} M_{\mathrm{sh}}$. However, it raises the question why such high Peierls barriers do not make themselves felt in the critical shear stress at temperatures well below the temperatures of the Bordoni and Niblett-Wilks peaks.

In the present writer's opinion there are two possible answers to the preceding question:

(a) Because of the interaction with other imperfections such as surfaces, impurities and, presumably more important, other dislocations (respectively their stress fields) the fraction of dislocations that ever reach the situation described in the preceding paragraph is small.

(b) At low temperatures quantum processes become important and permit a sufficiently large rate of kink-pair generation for the strain rate to be maintained even under moderate stresses.

In the 1950s and 1960s neither one of these answers was generally accepted. This led to quite a few attempts to explain the Bordoni relaxation by other models. In retrospect it can be said that none of them came anywhere near to accounting for the experimental facts as well as the kink-pair generation model. On the other hand, the refined experiments of $\mathrm{G}$. Alefeld and his associates and of the groups in Lausanne and 
Lyon gave further strong support to the basic correctness of the kink-pair generation model. This work has been adequately described in several reviews (see, e.g., [25]).

The mechanical relaxation due to kink-pair formation should occur also in crystals other than the facecentred cubic metals. In 1971 Seeger and Sesták [26] proposed that the so-called alpha and gamma relaxations of plastically deformed high-purity body-centred cubic (bcc) metals, which had been studied in detail by Chambers [27] and others, had an explanation analogous to that of the Bordoni relaxation in fcc metals. Specifically, they suggested that the gamma relaxation, which in metals such as $\mathrm{Nb}$, $\mathrm{Ta}$, or $\alpha-\mathrm{Fe}$ occurs near or somewhat above room temperature (i.e., at much higher temperatures than the Bordoni relaxation in, say, the noble metals) is caused by the thermally activated formation of pairs of kinks of opposite signs in screw dislocations with Burgers vectors $a_{0}\langle 111\rangle / 2\left(a_{0}=\right.$ edge length of the elementary cube).

High-purity bcc metals differ radically from high-purity fcc metals in that (among other things) below a so-called knee temperature $T_{\mathrm{K}}$ (which in the refractory bcc metals lies somewhat above room temperature) the flow stress increases rapidly with decreasing temperature and increasing strain rate. After long controversies, in the early 1970s it had become clear that this was an intrinsic property of the refractory bcc metals. It was ascribed to the sessile nature of the $a_{0}\langle 111\rangle / 2$ screw dislocations. In 1981 the present author suggested that the thermally activated formation of kink pairs on these dislocations accounts adequately for the temperature dependence of the flow stress of bcc metals below $T_{\mathrm{K}}$ [28]. This was found to be the case in $\mathrm{Nb}$, Ta, Mo, and $\alpha$-Fe [29, 30], thus supporting not only the kink-pair interpretation of the gamma relaxation in the refractory bec metals but indirectly also that of the Bordoni relaxation in the close-packed metals.

In Sect. 2 the kink-pair theory of the flow stress will be compared with the particlarly well-studied case of ultra-high purity Mo [30]. The excellent agreement between experiment and theory allows us to develop an atomistic picture of the core properties of the screw dislocations in the refractory bcc metals (Sect. 3). This will be seen to be the key for the understanding of the specific softening effects of atomic defects in bcc metals (Sect. 4).

\section{THE TEMPERATURE AND STRAIN-RATE DEPENDENCE OF THE FLOW STRESS OF HIGH-PURITY BCC METALS}

\subsection{Experiments}

Recently, L.Hollang [30] has performed very extensive measurements on ultra-high-purity Mo crystals. From starting material that had been purified and characterized in the Institut für Metallphysik und Reinstmetalle of the former Deutsche Akademie der Wissenschaften zu Berlin, Dresden (cf., e.g., the recent review by A. Köthe [31]), single-crystal rods of about $200 \mathrm{~mm}$ in length and $5 \mathrm{~mm}$ in diameter with residual resistivity ratios between of $1 \cdot 10^{5}$ and $4 \cdot 10^{5}$ were grown in the Institut für Festkörper- und Werkstofforschung Dresden e.V. From them Hollang prepared $\langle 941\rangle$-orientated specimens with circular cross-sections for either tensile tests (gauge length $10 \mathrm{~mm}$, diameters between $0.7 \mathrm{~mm}$ for low-temperature measurements and $1.1 \mathrm{~mm}$ for high-temperature measurements) or cyclic-deformation experiments (gauge length $10 \mathrm{~mm}$, diameter $4 \mathrm{~mm}$ ). The main substitutional impurity was tungsten (about $1 \mathrm{ppm}$ ). Interstitial impurities were below the limit of detectability; from the residual resistivity ratio Hollang estimated that their total concentration did not exceed $20 \mathrm{ppb}$.

Using the equipment of Brunner and Diehl [32] up to $320 \mathrm{~K}$ and a standard Zwick testing machine at higher temperatures, Hollang measured stress-strain curves in tension at a plastic strain rate of $\dot{\varepsilon}_{\mathrm{pl}}:=\mathrm{d} \varepsilon_{\mathrm{pl}} / \mathrm{d} t=8 \times 10^{-4} \mathrm{~s}^{-1}$ at 31 different temperatures between $4.2 \mathrm{~K}$ and $460 \mathrm{~K}$. The selection of stressstrain curves in Fig. 1 shows, over this temperature range not only the critical shear stress but alsothe shapes of the stress-strain curves varied considerably. As indicated in Fig. 1, at various plastic strains $\varepsilon_{\mathrm{pl}}$ the straining was interrupted for measurements of the stress relaxation at constant temperature. 
The cyclic-deformation experiments followed the procedures of Ackermann et al. [33], Werner [34], and Holzwarth [35] with slight modifications. The cyclic-deformation technique has the advantage that a complete set of flow-stress data covering a wide range of temperatures (in Hollang's work from $125 \mathrm{~K}$ to 460 $\mathrm{K}$, with reduced accuracy up to $530 \mathrm{~K}$ ) and plastic strain rates $\mid \dot{\varepsilon}_{\text {pl }}\left(\right.$ from $5.9 \cdot 10^{-7}$ to $1.7 \cdot 10^{-3}$ ) can be obtained from measurements on one single crystal, thus avoiding the reproducibility problems connected with the use of different samples in more standard measuring procedures. This is made possible by "preconditioning" the specimen by subjecting it at an elevated temperature $T_{\text {pre }}$ to cyclic deformation at constant plastic strain rate $\left|\dot{\varepsilon}_{\mathrm{pl}}\right|$ and strain amplitude $\hat{\varepsilon}_{\mathrm{pl}}$ until the maximum resolved shear stress, $\sigma_{\mathrm{max}}$, becomes independent of the number of cycles. (In the work on Mo reported here $\left|\dot{\varepsilon}_{\text {pl }}\right|=3.3 \cdot 10^{-5} \mathrm{~s}^{-1}$ and $\hat{\varepsilon}_{\mathrm{pl}}=1 \cdot 10^{-3}$.)

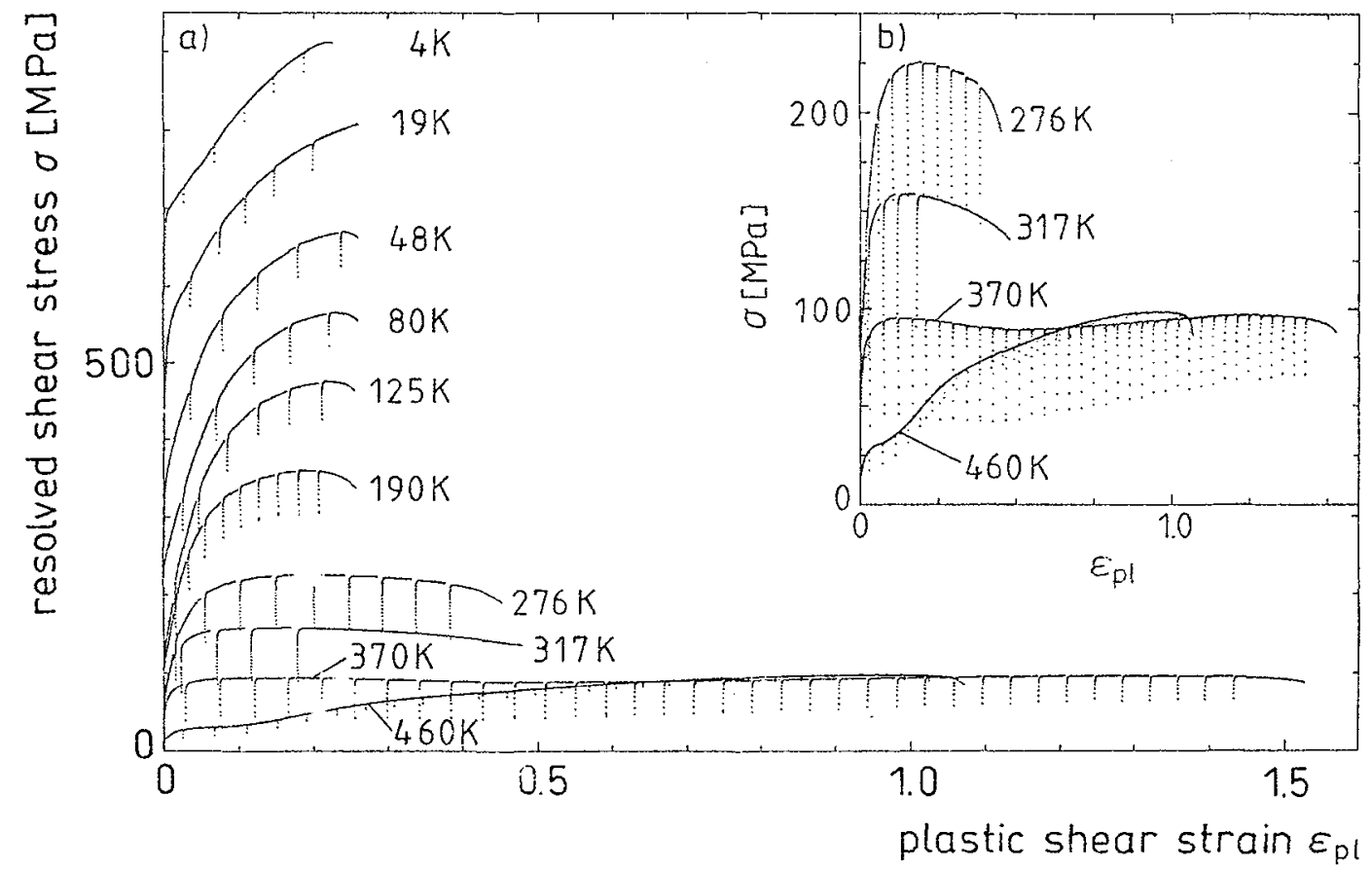

Figure 1: Stress-strain curves in tension of $\langle 941\rangle$-orientated single Mo crystal rods of ultra-high purity at different temperatures. Resolved shear stress $\sigma$ and plastic strain $\varepsilon_{\mathrm{pl}}$ were calculated for ( 101$)$ [111] glide; the plastic strain rate was $\dot{\varepsilon}_{\mathrm{pl}}=8.6 \times 10^{-4} \mathrm{~s}^{-1}$. The tensile tests were repeatedly internupted for stress relaxation and subsequent reloading. a) Selection of measured curves covering the entire temperature range investigated. b) Stress-strain curves between $276 \mathrm{~K}$ and $460 \mathrm{~K}$. Note the exceptional behaviour of the $370 \mathrm{~K}$ curve.

In his cyclic deformation experiments on ultra-high-purity Mo, Hollang [30] used $T_{\text {pre }}=530 \mathrm{~K}$. At this preconditioning temperature, the saturation stress $\sigma_{\text {sat }}=54 \mathrm{MPa}$ after about $5 \cdot 10^{3}$ cycles was reached. The microstructure obtained in this way proved to be very stable indeed. Stress-strain curves measured again at $530 \mathrm{~K}$ with the same $\left|\dot{\varepsilon}_{\mathrm{pl}}\right|$ and $\hat{\varepsilon}_{\mathrm{pl}}$ after the specimens had gone through a substantial number of measuring cycles at lower temperatures agreed within experimental error with the previous ones. If there was a slight deviation, the "saturation state" could quickly be restored by repeated cycling at the preconditioning temperature. 
After preconditioning at $T_{\text {pre }}=530 \mathrm{~K}$, Hollang [30] determined the flow stress $\sigma=\sigma\left(T, \dot{\varepsilon}_{\mathrm{pl}}\right)$ at 15 different strain rates, covering the range $5.9 \cdot 10^{-7} \leq\left|\dot{\varepsilon}_{\mathrm{pl}}\right| \leq 1.7 \cdot 10^{-3}$, at 5 different temperatures $5 \mathrm{~K}$ apart, and then preconditioned the specimen again under the conditions described above. Then the measuring programme was continued at a further set of temperatures under otherwise equal conditions. In a modification of the procedure used in the earlier work $[33,34]$ the strain-rate dependence of the flow stress was obtained by starting each deformation cycle at the so-called basic strain rate $\left|\dot{\varepsilon}_{\mathrm{pl}}\right|=3.3 \cdot 10^{-5} \mathrm{~s}^{-1}$ and then switching to the desired strain rate well before $\left|\hat{\varepsilon}_{\text {pl }}\right|$ was reached, where the flow stress $\sigma$ was determined. Compared to measurements of complete stress-strain cycles at fixed $\mid \dot{\varepsilon}_{\text {pll }}$, this procedure saved time and prolonged specimen life. The specimen failed at $120 \mathrm{~K}$ by brittle fracture after $\mathrm{N}=9 \cdot 10^{3}$ cycles without a harbinger variation of the shape of the hysteresis loop. This corresponded to a cumulative shear strain $\varepsilon_{\mathrm{pl}, \mathrm{cum}}=4 N \hat{\varepsilon}_{\mathrm{pl}}=36$ !

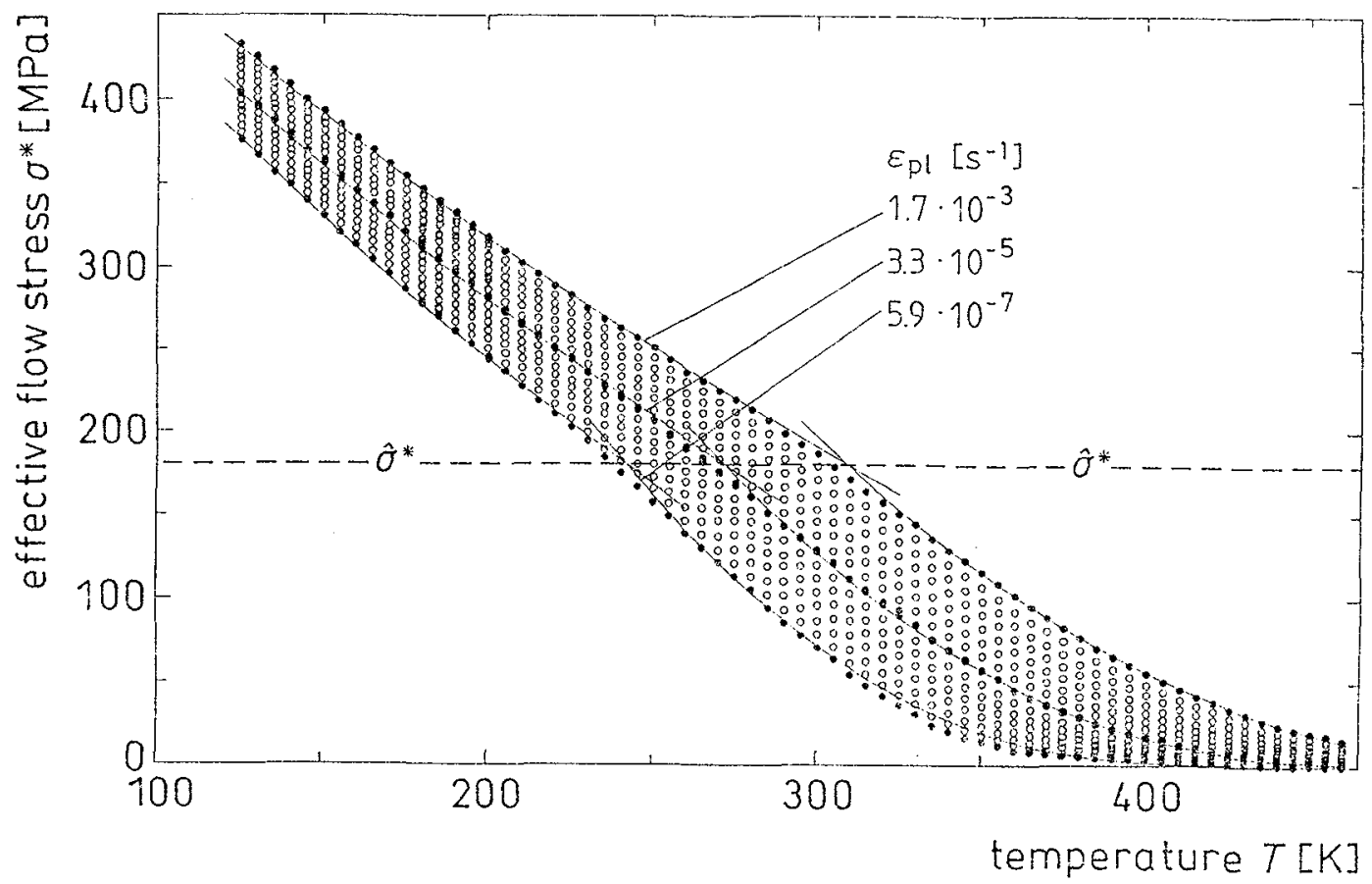

Figure 2: Temperature and strain-rate dependence of the effective flow stress $\sigma^{*}$ of an ultra-high-purity Mo crystal rod of $\langle 941\rangle$ orientation as measured by the cyclic deformation technique (see text). Resolved shear stress $\sigma^{*}$ and strain rate $\left|\dot{\varepsilon}_{\text {pl }}\right|$ refer to ( $\overline{1} 01)[111]$ glide. In units of $\mathrm{s}^{-1}$ the plastic strain rates $\left|\dot{\varepsilon}_{\mathrm{pl}}\right|$ increase from bottom to top in the sequence $5.9 \times 10^{-7}, 1.0 \times 10^{-6}$, $1.7 \times 10^{-6}, 3.3 \times 10^{-6}, 5.9 \times 10^{-6}, \ldots \ldots . .1 .0 \times 10^{-3}, 1.7 \times 10^{-3}$. The measurements at the "basic strain rate" $\left|\dot{\varepsilon}_{\mathrm{pl}}\right|=3.3 \times 10^{-5}$ (marked by full circles) were carried out twice and thus give an indication of the reproducibility. 
Fig. 2 shows the temperature dependence of the flow stress between $125 \mathrm{~K}$ and $460 \mathrm{~K}$ as determined by the procedure just outlined, with the strain rate $\left|\dot{\varepsilon}_{\text {pl }}\right|$ as parameter. At temperatures of $450 \mathrm{~K}$ or higher the maximum resolved shear stress (for glide on $\{110\}$ ) measured at the lowest strain rates assumed the strain-rate-independent value $\sigma_{\mathrm{M}}=53.3 \mathrm{MPa}$. This strain-rate independence indicated that under these conditions thermally activated processes no longer contributed to the flow stress. As will be argued in more detail below, $\sigma_{M}$ is believed to be associated with the long-range strains built up during the deformation leading to saturation; hence its temperature dependence should be entirely due to that of the elastic moduli, as indicated by the subscript $\mathrm{M}$. However, since in the present case the temperature variation of $\sigma_{M}$ is small compared with that of $\sigma$, we may treat $\sigma_{M}$ as strain-rate and temperatureindependent. The so-called effective stress

$$
\sigma^{*}=\sigma\left(T, \dot{\varepsilon}_{\mathrm{pl}}\right)-\sigma_{\mathrm{M}}
$$

which is the key quantity in the theory to be discussed below, is shown as the ordinate of Fig. 2 .

Fig. 2 is by far the most detailed plot of its kind so far obtained by the cyclic-deformation technique (based on more than thousand stress-strain curves measured at 68 different temperatures!). Otherwise, it is similar to the plots obtained in the earlier work on $\mathrm{Nb}[33,35]$, Ta [34, 33], Mo [36], and $\alpha-\mathrm{Fe}[36]$. All these results may be quantitatively interpreted by the model to be described in the following subsection.

\subsection{Theory}

The prescribed plastic strain rate $\dot{\varepsilon}_{\mathrm{pl}}$ is assumed to be maintained by one type of dislocations only, namely screw dislocations with Burgers vector $\boldsymbol{b}=a_{0}\langle 111\rangle / 2\left(a_{0}=\right.$ edge length of the elementary cube; in Mo $a_{0}=0.315 \mathrm{~nm}$ ). This is in agreement with both in-situ and post-deformation transmission electron microscopy. The plastic strain-rate may be written as

$$
\dot{\varepsilon}_{\mathrm{pl}}=b N_{\mathrm{d}} \bar{v}_{\mathrm{d}}
$$

where

$$
\bar{v}_{\mathrm{d}}=\bar{v}_{\mathrm{d}}\left(T, \sigma^{*}\right)
$$

is the mean dislocation velocity and $N_{\mathrm{d}}$ the density of the screw dislocations that maintain the strain rate. $\bar{v}_{\mathrm{d}}$ is assumed to depend on two independent variables, viz. the temperature $T$ and the effective flow stress $\sigma^{*}$ that assists the dislocations in overcoming localized barriers by thermal activation.

In the presence of long-range stress fields opposing the dislocation motion, $\sigma^{*}$ is less than the applied resolved shear stress $\sigma$. We make the specific assumption [37]

$$
\sigma\left(T, \dot{\varepsilon}_{\mathrm{pl}}\right)=\sigma_{\mathrm{M}}+\sigma^{*}\left(T, \dot{\varepsilon}_{\mathrm{pl}}\right)
$$

with $\sigma_{\mathrm{M}}$ not explicitly depending on $T$ and $\dot{\varepsilon}_{\mathrm{pl}}$.

Eq. (4) is completely equivalent to (1). The physical picture behind this assumption is (i) that $\sigma_{M}(>0)$ is due to the long-range stress fields of the dislocations in the neighbourhood of those dislocations whose motion we are considering and (ii) that the applied stress required to maintain the prescribed strain rate is determined by those dislocations which have to overcome the long-range stresses and local obstacles 
simultaneously. The non-dependence of $\sigma_{\mathrm{M}}$ on $\dot{\varepsilon}_{\mathrm{pl}}$ reflects the fact that thermal fluctuations are ineffective in assisting dislocations in overcoming the barriers due to long-range stress fields; nevertheless, if these stress fields are due to dislocations there will be a weak implicit dependence of $\sigma_{M}$ on temperature because of the temperature dependence of the elastic moduli $M_{\mathrm{ik}}$. The strong temperature and strain-rate dependence of $\sigma^{*}$ must therefore be attributed to obstacles to the dislocation motion that can be overcome by thermal fluctuations. In ultra-pure materials such as that used in the work of Hollang [30] impurities are unimportant; hence the obstacles have to be intrinsic. This means that their origin must lie in the discreteness of the crystal lattice; i.e., in the Peierls barriers referred to in Sect. 1. These are overcome by the stress-assisted thermally activated formation of kink pairs on the dislocations.

The extension of (4) to kink-pair formation as the rate-controlling process is a very plausible hypothesis. It may be tested experimentally at $\sigma^{*} \approx \sigma_{M}$, where it is most critical. At low strain rates there are indeed small differences between experiments and theory in the regime $\sigma^{*} \leq \sigma_{M}$ that might have partly to do with deviations from (4). We note, however, that the theory of kink-pair formation to be outlined presently does not depend on the validity of (4).

The key quantity in the theory of kink-pair formation is the net rate of kink-pair formation per unit dislocation length, $\Gamma_{\mathbf{k}}$. Its relationship to $\bar{v}_{\mathrm{d}}$ is obtained by considering the stationary state, in which the rate at which kinks disappear or become immobilized is exactly compensated by the generation of kink pairs. Kinks may annihilate when they meet kinks of opposite sign on the same dislocation line or may become immobilized when they are trapped by obstacles which they cannot overcome. Which of these two mechanisms is dominant depends on whether the mean distance between kinks of opposite sign, $\bar{x}_{\mathrm{k}}$, is larger or smaller than the mean distance between the obstacles, $\bar{L}$. In general the obstacles may either be foreign atoms or special configurations on the dislocation lines that have been created during the plastic deformation. Examples of the latter kind are dislocation nodes or immobile long jogs. In the cyclic deformation experiments the density of dislocations and hence that of the obstacles to kink motion is so high that $\bar{L}<<\vec{x}_{\mathbf{k}}{ }^{1}$. Then the relationship between the mean dislocation velocity and the net rate of kinkpair formation takes the simple form

$$
\bar{v}_{\mathrm{d}}=a \Gamma_{\mathrm{k}}\left(\sigma^{*}, T\right) \bar{L}
$$

where $a$ denotes the distance of the Peierls valleys connected by the kinks (in the following called kink height).

The calculation of the kink-pair formation rate $\Gamma_{\mathrm{k}}$ has been treated in detail elsewhere [38]. It is based on five main ideas:

(i) The motion of a dislocation lying approximately parallel to a Peierls-valley direction and therefore containing well-defined kinks may be decomposed into five different classes of degrees of freedom. These five classes, which may be treated as independent of each other, are:

${ }^{1}$ This may be seen as follows, using Hollang's [30] results on ultra-high-purity Mo as example. At high temperatures $\bar{x}_{\mathrm{k}}$ is the inverse of the linear equilibrium density of kinks of one sign, $\rho \underset{\mathrm{k}}{\mathrm{eq}}=\left(2 \pi m_{\mathrm{k}} k_{\mathrm{B}} T / h^{2}\right)^{1 / 2}$ $\exp \left(S_{\mathrm{k}} / k_{\mathrm{B}}\right) \exp (-H \mathrm{k} / k \mathrm{~B} T)$ [38]. Here $k_{\mathrm{B}}$ denotes Boltzmann's constant, $h$ Planck's constant, $S_{\mathrm{k}}$ and $H_{\mathrm{k}}$ the entropy and enthalpy of kink formation, and $m_{\mathrm{k}}$ the so-called kink mass. For $T=460 \mathrm{~K}$ we find with $H_{\mathrm{k}}=0.63$ $\mathrm{eV}$ and $m_{\mathrm{k}}=7.7 \cdot 10^{-27} \mathrm{~kg}$ the value $\exp \left(S_{\mathrm{k}} / k_{\mathrm{B}}\right) / \rho_{\mathrm{k}}^{\mathrm{eq}}=3.4 \times 10^{-4} \mathrm{~m}$. The parameter $N_{\mathrm{d}} \bar{L}$, where $N_{\mathrm{d}}$ denotes the density of mobile dislocations, has been determined as $7.2 \cdot 10^{6} \mathrm{~m}^{-1}$. Since $\exp \left(S_{\mathrm{K}} / k_{\mathrm{B}}\right)$ is expected to be of the order of magnitude unity and since in heavily fatigued specimens $N_{\mathrm{d}}$ presumably exceeds $10^{12} \mathrm{~m}^{-2}$, in the temperature range $T \leq 460 \mathrm{~K}$ the inequality $\bar{L} \geq \bar{x}_{\mathrm{k}}$ should hold. However, if the experiments are extended to substantially higher temperatures, this inequality might be reversed. As far as the experiments on ultra-highpurity Mo are concerned, in view of the low concentration of foreign interstitital atoms we may be fairly confident that the obstacles limiting $\bar{L}$ are not the foreign atoms. 
(a) The motions of the elastic continuum in which the dislocation is embedded. They may be described by "ordinary" (i.e., linearized) elasticity. The quanta of the "elementary excitations" connected with these degrees of freedom are the well-known acoustical phonons. Since these possess a linear dispersion relation, their rest mass is zero. In the following we call them light (or massless) phonons.

(b) The small-amplitude vibrational motions of the dislocation line in its Peierls valleys. These may be described by linear equations of motion but now, in contrast to (a), the frequency associated with zero wave-number is finite, namely equal to the vibrational frequency $v_{0}$ of a rigid dislocation in a Peierls valley. This means that the elementary excitations associated with these motions possess finite rest mass $m_{0}$. We call them heavy (or massive) phonons. The velocity $c_{\mathrm{d}}$ with which small short-wavelength perturbations propagate along a dislocation line in its Peierls valley plays the rôle of limiting velocity analogous to the velocity of light in the special theory of relativity.

(c) The translational motion of individual kinks.

(d) The translational motion of pairs of kinks of opposite sign (so-called kink-antikink pairs, or briefly, kink pairs).

(e) The relative motion of kink-antikink pairs along the dislocations.

(ii) The subsystem comprising the degrees of freedom (c) and (d) possesses (at least to a good approximation) solitonic properties [38]. This means that we may ascribe particle properties to the kinks (position, mass $m_{\mathrm{k}}$, mobility $\mu_{\mathrm{k}}$, diffusivity $D_{\mathrm{k}}$, momentum $p_{\mathrm{k}}$ ).

(iii) Since solitons do not exchange energy when colliding with each other, within the framework of statistical mechanics they may be treated as a gas of non-interacting particles [38 ]. The treatment of kinks as non-interacting particles and therefore as "soliton gas" should be an excellent approximation for the translational degrees of freedom (c and d) but is clearly not allowed for the remaining degree of freedom (e).

(iv) Degree of freedom (e) may be treated as the drift of a fictitious particle of mass $m_{1}=m_{\mathbf{k}} / 2$ (the reduced mass of two kinks of mass $\left.m_{\mathrm{k}}\right)$ and the mobility $\mu_{\mathrm{k}}=2 m_{\mathrm{k}}$ in a static potential $W\left(q_{1}, \sigma^{*}\right)$ that depends on the separation $q_{1}$ between kinks and antikinks, and which contains the effective stress $\sigma^{*}$ as a parameter [38]. This drift is assumed to be well described by a one-dimensional Klein-Kramers equation. For large separations $q_{1}$ the interaction between the kinks and the antikinks is mediated by the massless phonons of degree of freedom (a). In analogy to the electrostatic interaction, mediated by photons, it must therefore be coulombic. The force exerted by the effective resolved shear stress $\sigma^{*}$ on a kink of height $a$ in a dislocation of strength $b$ is $a b \sigma^{*}$. Hence for large enough $q_{1}$

$$
W\left(q_{1} ; \sigma^{*}\right)=a b q_{1} \sigma^{*}+a^{2} \gamma_{0} / 2 q_{1}
$$

holds. The "elastic charge" of the kinks appearing in (6), $a \sqrt{\gamma_{0} / 2}$, follows from a detailed calculation which shows that $\gamma_{0}$ is the prelogarithmic factor of the line-tension $\gamma_{\mathrm{d}}$ of the dislocations. $\gamma_{0}$ is proportional to $b^{2}$; the proportionality factor is determined entirely by the second-order elastic constants and may thus be calculated unambigously. For a screw dislocation in an elastically isotropic material it is given by $b^{2} M_{\mathrm{sh}}(2+v) / 8 \pi(1-v)$, where $M_{\mathrm{sh}}$ is the shear modulus and $v$ Poisson's ratio.

(v) Eq. (6) fails at small separations. The assumption is made that if this is the case, the elastic interaction between different parts of a dislocation line may be described by the so-called line-tension model. It makes two basic assumptions, viz. $(\alpha)$ that the self-interaction of a dislocation line may be taken into account in terms of a dislocation line tension $\gamma_{\mathrm{d}}$ and $(\beta)$ that a straight dislocation line that is displaced in its glide plane by an amount $u$ out of its Peierls valley under the action of an effective resolved shear stress $\sigma^{*}$ changes its energy per unit length, $E_{\mathrm{d}}$, by

$$
U_{\sigma}=U(u)-b \sigma^{*} u
$$


Assumption $(\alpha)$ implies that so-called non-local interactions between different segments of the dislocation line are neglected. A systematic derivation of the line-tension approximation allowing fully for elastic anisotropy has recently been given [39] on the basis of Kirchner's expression for the self-energy of a closed dislocation loop [40 ]. This treatment provides, for the first time, a straightforward procedure for the determination of the outer cut-off radius $R_{0}$ appearing in the ratio $\gamma_{\mathrm{d}} / \gamma_{0}=\ln R_{0} / r_{0}$, where $r_{0}$ is the inner cut-off radius. Whereas $r_{0}$ is determined by the properties of the dislocation core, $R_{0}$ is of the order of magnitude of the kink width $w$.

Provided the kink width $w$ is not too small, we may linearize the curvature term in the basic equation governing the line-tension model. This gives us

$$
m_{\mathrm{d}} \frac{\partial^{2} u}{\partial t^{2}}-\gamma_{\mathrm{d}} \frac{\partial^{2} u}{\partial z^{2}}-\frac{\mathrm{d} U_{\sigma}}{\mathrm{d} u}=0
$$

Here $t$ denotes the time, $z$ the spatial coordinate along the Peierls valley direction, and $u=u(z, t)$ the displacement of the dislocation line. For the deductions from (7) (kink formation enthalpy $H_{\mathrm{k}}$, kink-pair enthalpy $H_{\mathrm{kp}}\left(\sigma^{*}\right)$, vibration modes, etc.) the reader is referred to the literature [38].

\subsection{Comparison Theory-Experiment on High-purity Metals}

The cyclic-deformation experiments referred to above $[30,33,34,35]$ have confirmed the predictions of the theory to a very high degree. In this comparison the additional assumption was made that eq. (4) holds. One of the critical tests of the theory is that the temperature dependence of the flow stress should show a clearly discernable bend (the so-called "upper bend"; for the "lower bend" see Sect. 2.4) at the change-over from the validity of the "elastic-interaction model", described by eq. (6), to that of the linetension model described by eq. (8), at an effective flow stress $\hat{\sigma}^{*}$ that is virtually independent of the plastic strain rate $\dot{\varepsilon}_{\mathrm{pl}}$. As Fig. 2 shows, the cyclic deformation experiments on ultra-high-purity Mo by Hollang [30] have verified this prediction with high accuracy over the entire range of strain rates investigated ( 3.5 powers of ten). The less extensive cyclic deformation experiments on the other refractory bcc metals are in agreement with this prediction, too.

The physical meaning of $\hat{\sigma}^{*}$ is that of the effective shear stress $\sigma^{*}$ at which the kink-pair formation enthalpies $H_{\mathrm{kp}}\left(\sigma^{*}\right)$ as calculated by the line-tension model or by the elastic-interaction model intersect ${ }^{2}$. At this intersection the slope $\mathrm{d} H_{\mathrm{kp}}\left(\sigma^{*}\right) / \mathrm{d} \sigma^{*}$ predicted by the line-tension model is larger than that predicted by the elastic-interaction model $[28,38]$ by a factor between 1.59 and 1.69 . In his experiments on ultra-high purity Mo Hollang [30] found the ratio to be 1.63 , in excellent agreement with the prediction. Good agreement had also been obtained earlier on the other bcc metals investigated.

The full verification of the theoretical predictions for the upper bend gives strong support to the underlying hypothesis that the flow stress is determined by the same mechanism on both sides of $\hat{T}$. The bend can thus be understood entirely in terms of a rather sudden change-over in the regimes of validity of two different approximations to a general theory (not yet explicitly formulated). Deductions on the nature of the glide mechanism from measurements on one side of the bend apply on the other side as well.

\footnotetext{
${ }^{2}$ The effective flow stress of the "upper bend", $\hat{\sigma}^{*}$, was formerly denoted by $\bar{\sigma}^{*}$ (see, e.g., [38]). The change of notation to $\hat{\sigma}^{*}$ has been made for mnemonic reasons. The "circumflex accent" is used because at the upper bend the flow stress-vs.temperature curve is convex from above, whereas at the lower bend, to be designated by $\breve{\sigma}^{*}$, it is concave from above. The temperatures of the bends are denoted by $\hat{T}$ and $\breve{T}$, respectively. They are to be distinguished from the knee temperature $T_{\mathrm{K}}[28]$, which characterizes the temperature above which the contribution of the kink-pair formation mechanism to the flow stress becomes negligibly small.
} 
By fitting the temperature and strain-rate dependence of the flow-stress between $\hat{T}$ and $T_{\mathrm{K}}$ to the elastic-interaction model, the kink height $a$ may be deduced with high accuracy, provided the glide system and hence the so-called Schmid factor $\mu$ is known [28]. From a naive point of view one expects $a$ to be equal to the separation $a_{\{110\}}=2^{-1 / 2} a_{0}$ of the Peierls valleys on $\{110\}$ glide planes since $a_{\{110\}}$ is the shortest kink height in the bcc structure and since for a given height of the Peierls barrier the kink energy is proportional to $a$. With this expectation, based on energy considerations, the experiments on all refractory bcc metals investigated so far are in violent disagreement. There is excellent agreement, however, with $a=a_{\{211\}}$, where $a_{\{211\}}=(3 / 2)^{1 / 2} a_{0}$ is the separation of the Peierls valleys on the $\{211\}$ glide planes. E.g., on $\langle 941\rangle$ Mo crystals Hollang [30] obtained $a=(4.5 \pm 0.1) \cdot 10^{-10} \mathrm{~m}$ (allowing for the Schmid factor $\mu=0.43$ of the $\{211\}$ plane with largest $\mu$ ). This value corresponds to $1.76 a_{\{110\}}$ and agrees thus within the rather small experimental error with the prediction $a_{\{110\}}=3^{1 / 2} a_{0}$ for $\{211\}$ glide planes. Similarly good agreement was found by Brunner and Diehl [41] in stress-relaxation experiments on $\alpha-\mathrm{Fe}$. Hollang [30] has shown that in Mo $a=a_{\{211\}}$ can also be deduced from a fit of the line-tension model to the cyclicdeformation data between $\hat{T}$ and the lowest temperature investigated by him $(125 \mathrm{~K})$, though with slightly reduced precision.

From the preceding results we may draw the definite conclusion that at intermediate temperatures the elementary slip steps in the refractory bec metals occurs on $\{211\}$ planes. There appears to be no straightforward way to verify this conclusion by an independent technique. The slip planes that are deduced from the change of the crystal orientation in uniaxial deformation experiments are close to the plane of maximum resolved shear stress (m.r.s.s. plane); this is the result of easy cross-slip of screw dislocations in the temperature range in question [42]. The slip lines are rather wavy, following on the average approximately the m.r.s.s. planes. These observations are compatible with the hypothesis of elementary $\{211\}$ slip steps and easy cross-slip but, of course, do not exclude other interpretations.

The situation is quite different at low temperatures, say at liquid $\mathrm{He}$ or liquid- $\mathrm{H}_{2}$ temperatures. Here the slip lines on the refractory bcc metals are straight. In a recent review by B. Šsták and the present writer it is concluded that they provide strong evidence for the predominant operation of $\{110\}$ slip planes at low temperatures. This means that there must be a change in the glide mechanism of the refractory bcc metals - either gradual or sudden - somewhere between liquid- $\mathrm{H}_{2}$ temperatures and the lowest temperatures investigated by the cyclic deformation technique. Such a change should show up in the temperature and strain-rate dependence of the flow stress.

\subsection{The Lower Bend}

Brunner and Diehl [43] have called attention to a non-uniformity in the flow stress of $\alpha$-Fe single crystals at about $100 \mathrm{~K}$. It is characterized by the fact that the extrapolation of the flow-stress-vs.temperature curve, $\sigma=\sigma(T)$, from the regime well below this temperature (called regime III by Brunner and Diehl [43]) to higher temperatures gives flow-stress values that lie significantly below those measured at temperatures above the non-uniformity. This entails a rather rapid variation of $2 \sigma / \mathrm{d} T$ taken at constant plastic strain rate $\dot{\varepsilon}_{\mathrm{pl}}$ over a fairly narrow temperature interval. We call this variation the "lower bend" in order to distinguish it from the "upper bend" discussed above.

In order to emphasize that at the "lower bend" the sign of the change in $\partial \sigma / \partial T$ is opposite to that of the "upper bend" (in other words: $\sigma(T)$ remains concave from above over the entire temperature range of the lower bend) we denote the temperature and the effective flow-stress at which the extrapolated slopes meet by $\breve{T}$ and $\breve{\sigma}^{* 3}$.

\footnotetext{
${ }^{3}$ Brunner and Diehl denote the regime between $\breve{T}$ and $\hat{T}$ as "regime II". Regime $\mathbf{I}$ in their notation lies between $\hat{T}$ and $T_{\mathrm{K}}$.
} 
In his study of ultra-high-purity Mo single crystals with $\langle 941\rangle$ axes by uniaxial tensile and stressrelaxation measurements, which covered the temperature range $4 \mathrm{~K}<T<460 \mathrm{~K}$, Hollang [30] found that at a plastic strain rate of about $\dot{\varepsilon}_{\mathrm{pl}}=8 \cdot 10^{-4} \mathrm{~s}^{-1}, \widetilde{T}$ varied between about $190 \mathrm{~K}$ at low plastic strains and about $100 \mathrm{~K}$ at large plastic strains ${ }^{4}$. As a function of uniaxial plastic strain $\varepsilon_{\mathrm{pl}}, \breve{T}$ varied over a wide range. Taking this in conjunction with the above discussion on the planes of the elementary slip steps, we conclude that the origin of the lower bend is radically different from that of the upper bend.

The experimental facts discussed in the preceding paragraphs allow several conclusions to be drawn rather directly.

(1) We may exclude the possibility that the two slip mechanisms that dominate below or above $\breve{T}$ act in parallel and that they coexist in the temperature range in question. This follows simply from the fact that on both sides of $\breve{T}$ the suppressed slip mechanism would require a lower applied shear stress to maintain the prescribed strain rate than the mechanism actually operating.

(2) From (1) it follows that the lower bend cannot be accounted for by assuming the co-existence of two different types of dislocations that both contribute significantly to the strain rate. We conclude that slip is carried by the same dislocations above and below $\breve{T}$. This is in agreement with the evidence from electron microscopy, according to which the $a_{0}\langle 111\rangle / 2$ screw dislocations dominate from the lowest temperatures up to $T_{\mathrm{K}}$.

(3) Since the long-range stress field of screw dislocations is entirely determined by their Burgers vector (in this case $\mathbf{b}=a_{0}\langle 111\rangle / 2$ ) and since it can therefore not show large variations over a narrow temperature interval, the change in the elementary slip step at $\breve{T}$ must have its origin in changes in the core of the $a_{0}\langle 111\rangle / 2$ screw dislocations.

(4) If conclusion (3) is accepted, we have to ask the question whether the change-over in the core structure of the screw dislocations occurs because the low-temperature core configuration can no longer exist above $\widetilde{T}$ (in other words: because at $\breve{T}$ an order-disorder transition in the dislocation core takes place) or whether we are dealing with a first-order transition. In the latter case the transition takes place when the free dislocation line enthalpies of the two configurations,

$$
G_{\mathrm{d}}^{(j)}\left(\sigma^{*}, T\right)=H_{\mathrm{d}}^{(j)}\left(\sigma^{*}, T\right)-T S_{\mathrm{d}}^{(j)}\left(\sigma^{*}, T\right)
$$

become equal. In (9) the superscripts $j$ characterize the two configurations. Since we know that the two configurations are distinguished by the glide planes of their elementary slip steps, we shall use $j=\{110\}$ for the low-temperature configuration and $j=\{211\}$ for the high-temperature configuration. In the usual way, $H_{\mathrm{d}}$ refers to the dislocation line enthalpy, $S_{\mathrm{d}}$ to the dislocation line entropy.

As independent variables we have in (9) the temperature $T$ and the effective resolved shear stress $\sigma^{*}$ This, however, is only a shorthand notation for a more complicated situation. Since for a given uniaxial tensile stress the effective resolved shear stress $\sigma^{*}$ depends on the glide plane, it will in general be different for the two configurations. We assume this dependence, expressible in terms of the Schmid factor $\mu$, to be allowed for in the following. A further complication may arise from the well-known fact that the generalized Schmid law, which states that for the flow stress only one component of the applied stress tensor matters, namely the resolved shear stress in the glide system, is often violated in bcc metals. In this paper we shall not elaborate on this complication, but we should keep it in mind in discussions of detail.

Of the two alternatives considered in item (4), only the second one-a "first-order" transition in the dislocation core-is intuitively appealing. It appears difficult to imagine that the core of a dislocation in a

\footnotetext{
${ }^{4}$ The strain-rate dependence of $\breve{\sigma}$ has not been measured. It is planned to investigate it on high-purity W, for which $\breve{T}$ is expected to lie in the temperature range accessible to the Stuttgart cyclic-deformation facility.
} 
pure metal undergoes an order- disorder transition in such a way that in the disordered "high-temperature phase" a well-defined glide plane, viz. $\{211\}$, results.

We thus come to the conclusion (first drawn in [29]) that at $\breve{T}=\breve{T}\left(\sigma^{*}\right)$ a first-order transition in the core of the $a_{0}\langle 111\rangle / 2$ screw dislocation takes places and that the transition temperature is given by

$$
\breve{T}=\frac{H_{\mathrm{d}}^{\{211\}}\left(\sigma^{*}, T\right)-H_{\mathrm{d}}^{\{110\}}\left(\sigma^{*}, T\right)}{S_{\mathrm{d}}^{\{211\}}\left(\sigma^{*}, T\right)-S_{\mathrm{d}}^{\{110\}}\left(\sigma^{*}, T\right)} .
$$

The arguments leading to eq. (10) are so general that they do not require a detailed specification of the core configuration. They are thus independent of the discussions of Sect. 3.

\section{ATOMISTIC CONSIDERATIONS}

Before in Sect. 4 turning to the effects of foreign atoms ("impurities") and intrinsic atomic defects on the flow stress of refractory bcc metals, we insert a brief discussion of the atomistics of the dislocation configuration. A detailed account by B. Šesták and the present writer is being prepared and will give full references.



Figure 3: Projection of the bcc structure on the (111) plane. The numbering 1,2,3 denotes the different heights of the atoms along the [111] direction. The atoms surrounding the triangle centres A or B are located on spirals with opposite helicity.

Fig. 3 shows the projection of a bcc crystal along a [111] direction, the numbered circles representing the atom rows. These rows are crystal axes of threefold symmetry. The centres of the triangles are three-fold screw axes with different handedness (= helicity) in adjacent triangles. Let us now introduce an $a_{0}[111] / 2$ screw dislocation with a given helicity. As shown by $H$. Suzuki [44] on the basis of quite general arguments, the core contribution to its line energy is lower if the dislocation is located in the centre of a 
triangle with the same helicity as the dislocation than if it is located in one with the opposite helicity. Subsequent computations based on interatomic potentials have confirmed Suzuki's conclusion. Furthermore, they indicate that the dislocation positions in the centres of the triangles with the same helicity (in Fig. 4 marked by small open circles) are absolute minima if we consider the dislocation energy line, $E_{\mathrm{d}}(\boldsymbol{R})$, as a function of the two-dimensional position vector $\boldsymbol{R}$ in the $\{111\}$ plane.

By a general topological argument the periodic function $E_{\mathrm{d}}(\boldsymbol{R})$ must have at least three stationary points per elementary unit cell (in the present case a lozenge formed by two adjacent elementary triangles), namely in addition to the minimum just discussed a maximum and a saddle point. If the number of stationary points is minimal, for symmetry reasons the maxima and the saddle points of $E_{\mathrm{d}}(R)$ must coincide either with the three-fold axis (i.e., with the lattice rows) or with the centres of the triangles of opposite helicity. We denote the centres of the triangles with the same helicity as the screw dislocation by $\mathrm{A}$, those with the opposite helicity by $\mathrm{B}$, and the atom-row positions by $\mathrm{C}$. If by symmetry breaking (see below) the stationary points of $E_{\mathrm{d}}(R)$ move away from $\mathrm{A}, \mathrm{B}$ or $\mathrm{C}$, we mark the new positions by a dash.

A practical way of obtaining an estimate for $E_{\mathrm{d}}(\boldsymbol{R})$ with the minimum number of stationary points is to insert the displacement field of an $a_{0}\langle 111\rangle / 2$ screw dislocation as given by the linearized (anisotropic) theory of elasticity into the energy functional provided by a suitable model potential. Let us assume that this procedure predicts the saddle point of $E_{\mathrm{d}}$ to lie in the centre of the triangles with opposite helicity, i.e., in the $\mathrm{B}$ position. By the very definition of a saddle point, this is a configuration of unstable equilibrium. Thus, if the atomic positions in and near the dislocation core are allowed to relax, i.e., to take up positions which satisfy the equilibrium conditions for the model potential rather than those of linearized elasticity, the three-fold screw symmetry will be broken and new saddle point positions $B^{\prime}$ will emerge. There are two possibilities:



Figure 4: Insertion of a screw dislocation in an A triangle center with the same helicity. The saddle points resulting from the relaxation of the dislocation core are indicated. The possibilities (a) and (b) give rise to different Peierls potentials (bottom). 
(a) The new saddle points lie on the three $\{2 \overline{1} \overline{1}\}$ planes through the centre of the triangle. Then one obtains three saddle points $B^{\prime}$ per elementary cell as indicated in Fig. 4 by the full triangles.

(b) The new saddle points do not lie on the above-mentioned $\{2 \overline{1} 1\}$ planes. Then each of the original saddle points is replaced by six saddle points $B^{\prime}$, as indicated by the crosses in Fig. 4 .

Next we consider the glide of a screw dislocation from one position of minimum line energy to a neighbouring one, i.e., from one A position to the next. The path of lowest energy will pass through one [case (a)] or two [case (b)] saddle points, as indicated by the interrupted lines in Fig. 4. If we represent this by a "Peierls potential" $U(u)$ as a function of the projection $u_{\{110\}}$ of the instantaneous position of the dislocation line on the $(1 \overline{1} 0)$ plane, we obtain two different types of curves depending on whether case (a) or case (b) is realized (Fig. 4, bottom).

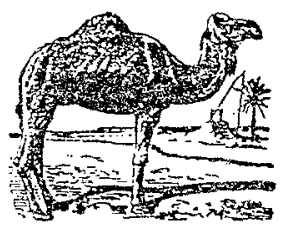

Arabian camel

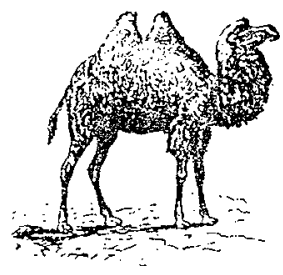

Bactrian camel

Figure 5: The two camel species (see text)

In the Japanese literature the "two-hump" Peierls potential of case (b) is often referred to as the "camel hump" potential. From a zoological point of view this may be misleading. There are two kinds of camels, the Arabian camel with one hump (camelus dromedarius) and the Bactrian camel (camelus bactrianus) with two humps (Fig.5). The Arabian camel corresponds to case (a), the Bactrian camel to case (b). The conclusion of the present paper depends only on the fact that the Peierls potential has the shape of a camel back, but not on whether it reminds us of the species camelus dromedarius or the species camelus bactrianus. The reader will note that the designations (a) and (b) in Fig. 4 have been chosen mnemonically.

The assumptions made so far imply that $E_{\mathrm{d}}(\boldsymbol{R})$ as calculated from the elasticity solution has an absolute maximum at $C$. Here we expect a particularly large effect if we allow the atomic positions to relax. $B$. Sesták and the present writer have studied this relaxation by means of a "balls and bonds" model of the bcc structure. It was found, in agreement with the conclusions of Schoeck [45], that the dislocation axis tends to move away from $\mathrm{C}$ on one of the $\{11 \overline{2}\}$ planes through $\mathrm{C}$ into one of the adjacent triangles with helicity opposite to that of the screw dislocation until a local minimum $\mathrm{C}^{\prime}$ is reached (in Fig. 6 marked by a cross in a small circle). In this configuration the dislocation has a well-defined slip plane (in the special case shown in Fig. 6 the $(11 \overline{2})$ plane). Peierls potential $U(u)$ for the dislocation displacement $u_{\{11 \overline{2}\}}$ on $(11 \overline{2})$ out of the equilibrium position $C^{\prime}$ goes through a maximum at a position $B^{\prime}$ which may be obtained by applying the relaxation process in a restricted way (see below) to the B position. In Fig. 6 this $\mathrm{B}^{\prime}$ position is marked by a full triangle in order to indicate that in our construction it has the same origin as the saddle points B or B' in Fig. 4.

The period of $U(u)$ in Fig. 6 is $a_{\{211\}}$, whereas in Fig. 4 it was $a_{\{110\}}=3^{-1 / 2} a_{\{211\}}$. In Fig. $4 U(u)$ is symmetric with respect to $u_{\{110\}}=0$ and $u_{\{110\}}=a_{\{110\}} / 2$; by contrast, the saw-toothed $U(u)$ of Fig. 6 has in general no symmetry other than the abovementioned periodicity. Generically, we may refer to it as "saw-tooth potential". 




Figure 6: Insertion of a screw dislocation along an atom row with subsequent relaxation of the dislocation core. The resulting Peierls potential for $\{211\}$ glide is indicated on the right-hand side.

When a screw dislocation moves on a $\{211\}$ plane from one $\mathrm{C}^{\prime}$ position to a neighbouring one, it necessarily passes through the centre of a triangle with the same helicity at its own, i.e., through an A position. According to our starting hypothesis, the A positions are absolute minima of the line-energy surface $E_{\mathrm{d}}(\boldsymbol{R})$. They should therefore also be minima if displacements on $\{211\}$ planes through A are considered. This is indicated by the parabola at the right-hand side of Fig. 6 . Why then does $U(u)$ lie far above this parabola? The key to the answer is that the Peierls potential $U(u)$ is not a section through the line-energy surface $E_{\mathrm{d}}(\boldsymbol{R})$. The Peierls potential has been introduced in eq. (7) in order to treat the shape of dislocation lines when they do not run parallel to the direction of a Peierls valley, e.g., if they form kinks or kink pairs. This means that the dislocations have an edge component of variable magnitude even if the direction of the Peierls valley happens to be that of the Burgers vector, as is the present case. This means that even for screw dislocations, $U(u)$ pertains to a well-defined glide plane. Hence the atomic relaxation in the dislocation core must be restricted accordingly. The A configuration with its three-fold screw symmetry cannot have a well-defined glide plane and must therefore be disregarded in the construction of the Peierls potential for $\{211\}$ glide.

On the other hand, it is just the fact that the fully relaxed A configuration does not have a well-defined glide plane that is responsible for the high Peierls barriers of screw dislocations in the refractory bcc metals compared to those in, say, face-centred cubic metals. In order to be able to glide on one of the $\{110\}$ planes, the core of the screw dislocations in the A configuration must undergo a major rearrangement with the accompanying expenditure of energy. By contrast, the $C^{\prime}$ configuration does have a well-defined glide plane; hence the energy difference between $\mathrm{B}^{\prime}$ and $\mathrm{C}^{\prime}$ in Fig. 6 should be distinctly 
smaller than the corresponding barrier in Fig.4. (Clearly, in these diagrammes the $U(u)$ profiles are not drawn to scale.)

Why is it then that the kink formation enthalpy $H_{\mathrm{k}}$ is much higher for $\{211\}$ elementary glide steps than for $\{110\}$ glide? According to Hollang [30], in $\mathrm{Mo}$ we have $2 H_{\mathrm{k}}=(1.27 \pm 0.02) \mathrm{eV}$ in the first case and $2 H_{\mathrm{k}}=(0.63 \pm 002) \mathrm{eV}$ in the second.

For a fixed shape of $U(u)$ and a given height of the Peierls barrier (in Fig. 6 equal to the energy difference between $\mathrm{B}^{\prime}$ and $\mathrm{C}^{\prime}$ ) the kink energy is proportional to the kink height $a$. In the present case this is not enough to account quantitatively for the ratio of about 2 between the kink formation enthalpies. This means that, in addition, we have to allow for the fact that the dislocation line energy in the $\mathrm{C}^{\prime}$ configuration is higher than in the A configuration.

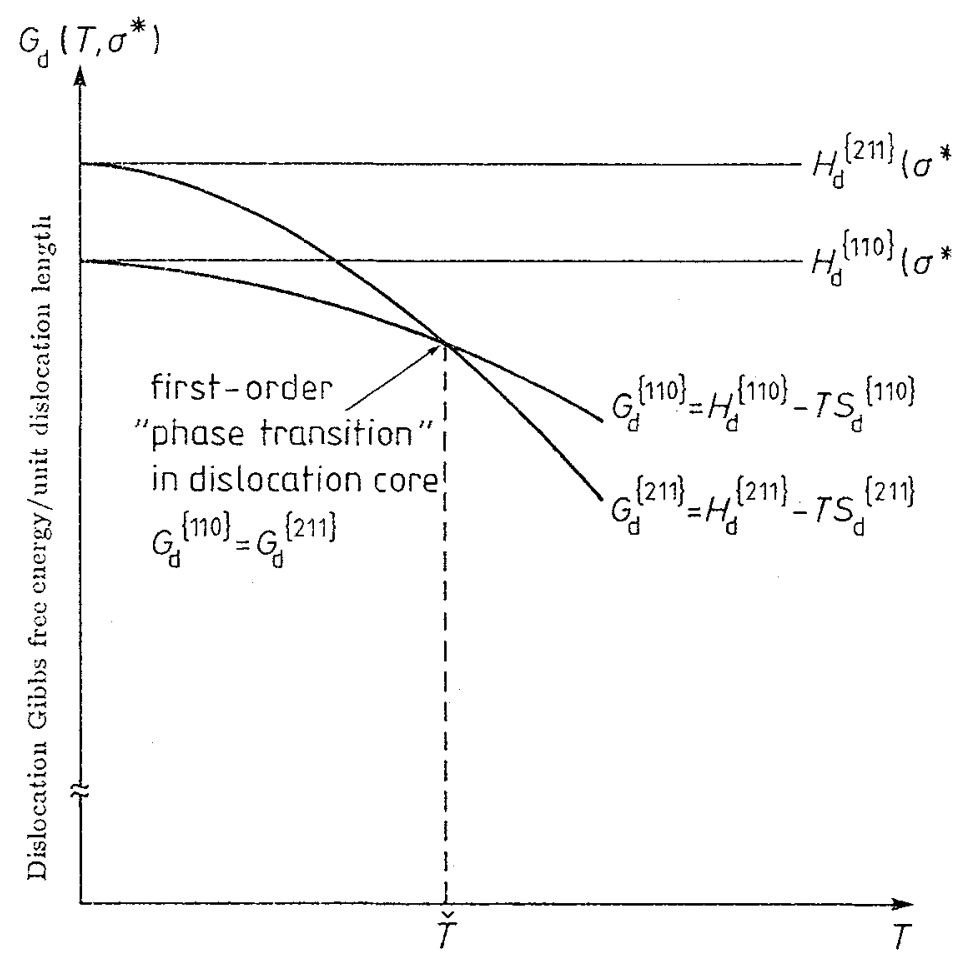

Figure 7: Cut through the proposed surface of the Gibbes free dislocation line energy $G_{\mathrm{d}}\left(T, \sigma^{*}\right)$ for $\mathrm{a}_{0}\langle 111\rangle / 2$ screw dislocations with either $\{110\}$ or $\{211\}$ glide planes. The cut follows the temperature variation $\sigma^{*}(T)$ of the effective flow stress for a given plastic strain rate $\dot{\varepsilon}_{\mathrm{pl}}$, hence it depennds on $\sigma^{*}$. The intersection of $G_{\mathrm{d}}^{\{110\}}$ and $G_{\mathrm{d}}^{\{211\}}$ gives the temperature $\breve{T}$ of the "lower band"

Qualitatively we may argue as indicated in Fig. 7. The dislocation line energy (energy per unit dislocation length) is substantially higher in the $\mathrm{C}^{\prime}$ configuration than in the A configuration. Since for $\{211\}$ glide in the "soft" direction $U(u)$ is flatter than $U(u)$ for $\{110\}$ glide, application of an effective resolved shear stress $\sigma^{*}$ favouring $\{211\}$ slip in the soft direction will reduce the difference $\Delta H\left(\sigma^{*}\right)$ between the $\{211\}$ and the $\{110\}$ configurations. At the same time, the $\{211\}$ Peierls potential becomes "softer" compared with the $\{110\}$ Peierls potential. Hence for the dislocation line entropies, $S_{\mathrm{d}}^{\{211\}}$ and $S_{\mathrm{d}}^{\{110\}}$, the 
inequality $S_{\mathrm{d}}^{\{211\}}>S_{\mathrm{d}}^{\{110\}}$ holds. As the temperature increases, this will lead to a change in sign of the difference between the Gibbs free line energies of the two configurations,

$$
\Delta G_{\mathrm{d}}=G^{\{211\}}\left(T, \sigma^{*}\right)-G^{\{110\}}\left(T, \sigma^{*}\right)
$$

with

$$
G_{\mathrm{d}}^{(j)}\left(T, \sigma^{*}\right)=H_{\mathrm{d}}^{(j)}\left(T, \sigma^{*}\right)-T S_{\mathrm{d}}^{(j)}\left(T, \sigma^{*}\right), j=\{211\},\{110\}
$$

According to our starting assumption $\Delta G_{\mathrm{d}}$ is positive at low temperatures. At the temperature at which $\Delta G_{\mathrm{d}}=0$ holds, the dislocation core will undergo a first-order phase transition. As was done earlier [29], we identify this temperature with the temperature $\breve{T}$ of the lower bend. However, the inequality $H_{\mathrm{d}}^{\{211\}}>H_{\mathrm{d}}^{\{110\}}$ continues to hold even above $\breve{T}$ (Fig. 7). This should give a contribution to the activation enthalpy for the $\{211\}$ glide steps that is large enough to account for the experimentally observed values.

\section{THE INFLUENCE OF ATOMIC DEFECTS ON THE FLOW STRESS}

The perturbation of the crystal structure accompanying the incorporation of atomic defects, be they intrinsic (e.g., vacancies or self-interstitials) or extrinsic (foreign atoms), interferes with the motion of dislocations. This leads to well-known and thoroughly studied hardening phenomena such as alloy hardening, irradiation hardening, and quench hardening. It was therefore a major surprise when, in the bcc metals, examples of alloy and irradiation softening were found [46]. To the best of our knowledge a truly convincing explanation for this phenomenon has not been given so far.

The first-order transition in the cores of $a_{0}\langle 111\rangle / 2$ screw dislocations offers a straight-forward explanation of the softening phenomena mentioned above. All we have to postulate is that certain atomic defects can reach the dislocation cores and cause them to "overheat", i.e., to shift the phase transition from the low-temperature $\{110\}$ configuration to the high-temperature $\{211\}$ configuration towards higher temperatures. Atomic defects that are mobile at $\breve{T}$ and can therefore reach the dislocation cores below the transition temperature are particularly good candidates for producing this overheating effect. This is born out by the experiments; the softening effects due to interstitial hydrogen and to intrinsic atomic defects created by irradiation have been clearly identified. In the latter case the softening is presumably due to those self-interstitials that begin to migrate freely in the so-called recovery stage $\mathrm{I}_{\mathrm{E}}$, the "crowdions" [47]. However, by suitable heat treatments other atomic defects, e.g. "heavy" interstitial foreign atoms such as carbon, nitrogen, and oxygen, can be incorporated in the cores of $a_{0}\langle 111\rangle / 2$ screw dislocations of the refractory bec metals, too, and can give rise to alloy softening.

A straightforward prediction following from the "overheating hypothesis" is that if there are no complicating effects such as hardening by one of the "usual" mechanisms, the temperature dependence of the flow stress, $\sigma=\sigma_{\mathrm{M}}+\sigma^{*}\left(T, \dot{\varepsilon}_{\mathrm{pl}}\right)$, in the "overheating regime" should follow the extrapolation of the low-temperature flow stress up to the temperature at which the overheating ends. There $\sigma^{*}\left(T, \dot{\varepsilon}_{\text {pl }}\right)$ should return to the temperature dependence of the flow stress in the absence of the softening effects.

Brunner and Diehl have investigated the influence of a range of nitrogen concentrations on the flow stress of $\alpha-\mathrm{Fe}$ [48]. Their results for the temperature variation of the flow stress of the alloy containing 330 at.ppm $\mathrm{N}$ follows the preceding prediction rather well (Fig.8). There is little influence of the alloying below $\breve{T} \approx 85 \mathrm{~K}$. Above that temperature the flow stress in the alloy follows the extrapolation of the lowtemperature flow stress up to about $165 \mathrm{~K}$. The flow-stress of pure $\alpha-\mathrm{Fe}$ is reached again at $275 \mathrm{~K}$. There 
is a slight indication in the data that above that temperature the nitrogen addition has a hardening effect. This is not surprising, since in this temperature regime the flow stress is so low that "normal" alloy hardening should indeed make itself felt.

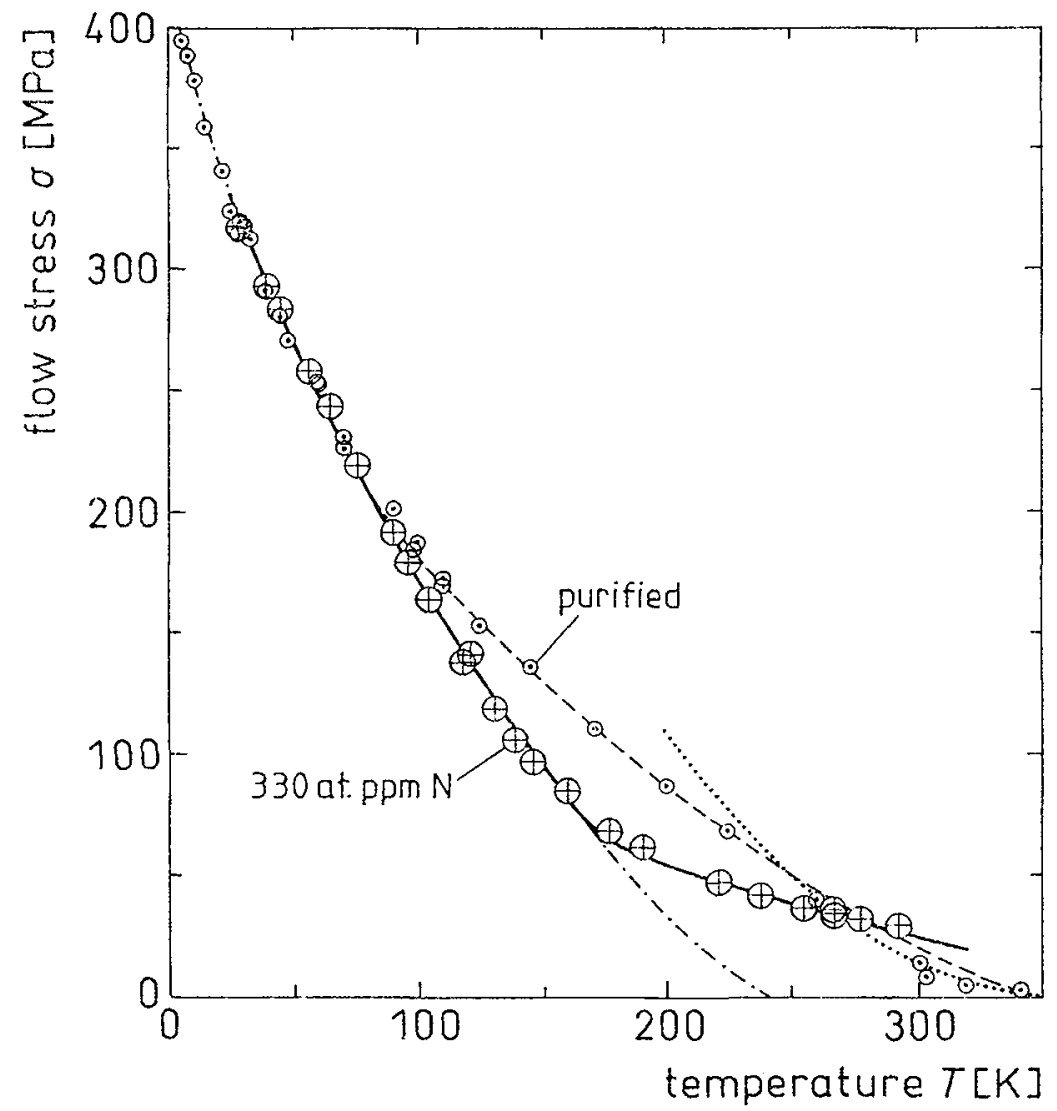

Figure 8: Temperature dependence of the flow stress of pure and $\mathrm{N}$-doped $\alpha$-Fe according to Brunner and Diehl [48]

In a series of papers Brunner, Diehl and their associates [49-51] have shown that the softening effects in $\alpha-F e$ due to intrinsic atomic defects are much more complicated than those due to foreign atoms. On the basis of flow-stress measurements on $\alpha$-Fe single crystals irradiated at liquid-nitrogen temperatures with $2.5 \mathrm{MeV}$ electrons, they distinguish between primary and secondary irradiation softening [50]. "Primary irradiation softening" is observed below $\widetilde{T}$ (i.e., in the temperature range that remained unaffected by doping with 330 at $\mathrm{ppm} \mathrm{N}$ ), provided the irradiated sample was not annealed at temperatures of about $145 \mathrm{~K}$ or above. If the samples are annealed in this temperature range but not above about $220 \mathrm{~K}$, the softening is confined to temperatures above the lower bend in the pure crystals. Although the available data are not as extensive as those obtained in N-doped crystals, this "secondary irradiation softening" resembles the alloy softening in every respect except for the fact that it recovers at about $220 \mathrm{~K}$. In particular, the temperature variation of the flow stress above $\breve{T}$ follows the extrapolation of the lowtemperature data. We may thus conclude that the "secondary irradiation softening" is due to the overheating of the transition from the $\{110\}$ configuration to the $\{211\}$ configuration of the screw dislocation core. The fact that the recovery temperature of the secondary irradiation softening (ca. $220 \mathrm{~K}$ ) coincides with the so-called recovery stage III suggests strongly that this overheating is caused by the atomic defects that anneal out in this recovery stage, viz. self-interstitials in the dumb-bell configuration $[47]$. 
The mechanism of primary irradiation softening is much more difficult to identify. Since this softening has been observed under conditions (electron irradiation at $78 \mathrm{~K}$ without subsequent warming up) at which there are no freely mobile intrinsic defects in $\alpha-\mathrm{Fe}$ (recovery stage $\mathrm{I}_{\mathrm{E}}$ occurs at much higher temperatures) it seems plausible that the softening effect is due to self-interstitials that have reached the dislocations as "dynamic crowdions" [52]. Since the recovery behaviour of primary and secondary irradiation softening is different, different defect configurations must be responsible for the two phenomena. A natural suggestion is that primary irradiation softening is caused by self-interstitials in the crowdion configuration. Although computer modelling has given some insight into possible interactions between crowdions and screw dislocations in bcc metals [53], it must be conceded that a truly convincing atomistic explanation for primary irradiation hardening has not yet emerged. We attribute this to some extent to the fact that the available experimental information is still rather limited.

\section{CONCLUSIONS}

The temperature and strain-rate dependent part of the flow stress of high-purity body-centred cubic metals is very well described by the theory of kink-pair formation on $a_{0}\langle 111\rangle / 2$ screw dislocations. The core of these dislocations may assume two different configurations, viz. one which allows glide on $\{110\}$ planes and another one that may glide on one specific $\{211\}$ plane. The transition from the lowtemperature $\{110\}$ configuration to the high-temperature $\{211\}$ configuration is a first-order transition and can therefore be "undercooled" or "overheated". Atomic defects interacting with the dislocation cores may cause "overheating". This leads to softening in the temperature range above the temperature $\breve{T}$ of the transition in the dislocation cores. The main features of this explanation have been verified experimentally for alloy softening [48] as well as irradiation softening [51].

\section{Acknowledgements}

The author wishes to express his sincere thanks to Dipl.-Phys. L. Hollang, Prof. W. Frank, and Drs. D. Brunner, J. Diehl, and B. Sesták for many valuable discussions and their help.

\section{References}

[1] Seeger A.,in: "Ultra-High-Purity Base Metals", UHPM-94, K. Abiko, K. Hirokawa, S. Takaki, Eds. (The Japan Institute of Metals, Sendai 1995) p. 27.

[2] Mott N.F., and Gurney R.W., Electronic Processes in Ionic Crystals (Oxford University Press, Oxford 1940).

[3] Schmid E., and Boas W., Kristallplastizität unter besonderer Berücksichtigung der Metalle, Springer, Berlin 1935.

[4] Boas W., and Schmid E., Z. Physik 54 (1929) 16.

[5] MacKenzie J.K., Ph.D. Thesis, University of Bristol, Bristol, 1949.

[6] Seeger A., "Kristallplastizität", in: Encyclopedia of Physics, Vol.VII/2 (S. Flügge, Ed.) Springer, Berlin 1958, Sect. 5.

[7] Meissner W., Polyani M., and Schmid E., Z. Physik 66 (1930) 477.

[8] Prandtl L., Z. Angew. Math. Mech. 8 (1928) 85.

[9] Zener C.N., Elasticity and Anelasticity of Metals, Chicago University Press, Chicago 1948.

[10] Dehlinger U., Ann. Phys. [5] 2 (1929) 749.

[11] Orowan E., Z. Physik 89 (1934) 634.

[12] Polyani M., Z. Physik 89 (1934) 660.

[13] Taylor G.I., Proc. Roy. Soc. Lond. 145 (1934) 362.

[14] Peierls R., Proc: Phys: Soc. Lond. 52 (1940) 34.

[15] Peierls R., in: Dislocation Dynamics (A.R. Rosenfield, G. T. Hahn, A. L. Bement, Jr., and R. I. Jaffee, Eds.), Mc Graw-Hill, New York 1968, p. xiii.

[16] Nabarro F.R.N., Proc. Phys. Soc. 59 (1947) 236.

[17] Dietze H.-D., Z. Physik 131 (1952) 156. 
[18] Frank F.C., and Read, W.T., Phys. Rev. 79 (1950) 722.

[19] Blewitt T.H., Coltman, R. R., and Redman J.K., Report on a Conference on Defects in Solids, held in Bristol 1954, The Physical Society, London 1965, p. 349.

[20] Seeger A., Theorie der Gitterfehlstellen, in: Encyclopedia of Physics (S. Flügge, Ed.), Vol. VII/1, Springer, Berlin etc. 1955, Sect. 72.

[21] Seeger A., Phil. Mag. 1 (1956) 651.

[22] Bordoni P.G., Ricerca Scient. 19 (1949) 851.

[23] Bordoni P.G., J. Acoust. Soc. Am. 26 (1954) 495.

[24] Niblett D. H., and Wilks J., Phil. Mag. 1 (1956) 415.

[25] Ritchie I.G., and Fantozzi, G., in: Dislocations in Solids, Vol. 9 (F. R. N. Nabarro, Ed.), Elsevier Science Publishers, Amsterdam 1992, 57.

[26] Seeger A., and Sesták, B., Scripta Metall. 5 (1971) 875.

[27] Chambers R.H., in: Physical Acoustics, Vol. III A (W. P. Mason, Ed.), Academic Press, New York and London 1966, 123.

[28] Seeger A., Z. Metallkde. 72 (1981) 369.

[29] Seeger A., in: Selected Topics on Condensed Matter Physics (W. E. Alnaser, Ed.), University of Bahrain 1993, ISBN 1870199 09X, p. 192.

[30] Hollang L., Dr. rer. nat. thesis, submitted to Universität Stuttgart, Stuttgart 1995.

[31] Köthe A., in: Ultra-High-Purity Base Metals (UHPM-94, K. Abiko, K. Hirokawa, and S. Takaki, Eds.), The Japan Institute of Metals, Sendai 1995, p. 291

[32] Brunner D., and Diehl J., Z. Metallkde 83 (1992) 2.

[33] Ackermann F., Mughrabi H., and Seeger A., Acta Metall. 31 (1983) 1353.

[34] Werner M., phys. stat. sol. (a) 104 (1987) 63.

[35] Holzwarth U., and Seeger A., in: Proceedings of the International Conference on the Strength of Materials (D. G. Brandon, R. Chaim, and A. Rosen, Eds.), Freund Publishing House Ltd. London 1991, Vol. 1, p. 577.

[36] Ackermann F., and Mughrabi H., unpublished work.

[37] Seeger A., Phil. Mag. 45 (1954) 771.

[38] Seeger A., in: Dislocations 1984, (P. Veyssière, L. Kubin, and J. Castaing, Eds.), Editions C.N.R.S. Paris 1984, p. 141.

[39] Hauser H.-M., Diplomarbeit Universität Stuttgart, 1995.

[40] Kirchner H. O. K., Phil Mag. 43 (1981) 1393.

[41] Brunner D., and Diehl J., phys. stat. sol (a) 124 (1991) 155.

[42] Šesták B., and Seeger A., Z. Metallkde. 69 (1978) 195, 355, 425.

[43] Brunner D. and Diehl J., phys. stat. sol. (a) 104 (1987) 145.

[44] Suzuki H., in: Dislocation Dynamics (A. R. Rosenfield, G.-T. Hahn, A.L. Bement, Jr., and R. I. Jaffee, Eds.) Mc Graw-Hill, New York etc. 1968, p. 679

[45] Schoeck G., Scripta Metall. 14 (1980) 983.

[46] Pink E., and Arsenault R.I., Progr. Mat. Science 24 (1979) 1.

[47] Frank W., and Seeger A., Materials Science Forum 15 - 18 (1987) 57.

[48] Brunner D., and Diehl J., J. Mater. Sci. Eng. A 164 (1993) 350.

[49] Aono, Y., E. Kuramoto, D. Brunner and Diehl, J., Materials Science Forum 15 - 18 (1987) 801.

[50] Aono Y., Brunner, D., and Diehl, J., in: Strength of Metals and Alloys (ICSMA 8, P. O. Kettunen, T. K. Lepistö, and M.E. Lehtonen, Eds.), Pergamon Press, Oxford 1988, Vol. 1, p. 509.

[51] Brunner D., and Diehl, J., in: Strength of Materials (ICSMA 10, K. Oikawa, K. Maruyama, S. Takeuchi, and M. Yamaguchi, Eds.), The Japan Institute of Metals, Sendai 1994, p. 141.

[52] Seeger A., in: Proc. 2nd UN Internat. Conf. on the Peaceful Uses of Atomic Energy, Vol. 6, Geneva 1958 , p. 250.

[53] Aono Y., Tsutsumi, T., and Kuramoto, E., in: Strength of Materials (ICSMA 10, K. Oikawa, K. Maruyama, S. Takeuchi, and M. Yamaguchi, Eds.), The Japan Institute of Metals, Sendai 1994, p.93 